\title{
Changes levels of myokines after aerobic training and resistance training in post-menopausal females with obesity
}

\section{Sunghwun Kang}

Kangwon National University

II Bong Park

Busan University of Foreign Studies

Seung-Taek Lim ( $\square$ limdotor@gmail.com )

Nasaret International Hospital https://orcid.org/0000-0002-0980-991X

Research article

Keywords: myokines, aerobic training, resistance training, obesity, women

Posted Date: March 6th, 2020

DOI: https://doi.org/10.21203/rs.3.rs-16256/v1

License: (a) (1) This work is licensed under a Creative Commons Attribution 4.0 International License.

Read Full License 


\section{Abstract}

Background To investigate changes in levels of myokines after regular aerobic training and resistance training in post- menopausal females with obesity.

Methods Among 41 post- menopausal obese females volunteered to participate in this study performed regular physical exercise training (aerobic exercise and resistance exercise). These participants were randomly assigned to one aerobic exercise group $(n=21)$ and one resistance exercise group $(n=20)$. The experimental group performed three days of aerobic exercise and resistance exercise per week (i.e., Monday, Wednesday, and Friday) each group. Body composition, physical fitness, and myokines were measured at baseline, 6 weeks, and 12 weeks.

Results Two-way within-factor ANOVA revealed group $\times$ time interaction for body mass index (BMI, $\mathrm{p}<$ $0.05)$. In the resistance exercise group, muscle endurance, power, and agility were significantly increased at 12 weeks than those at 6 weeks and baseline. In the aerobic exercise group, muscle strength, flexibility, muscle endurance, and agility were greater at 12 weeks than those at 6 weeks and baseline. Levels of IL$6, I L 15$, and BDNF were greater at 12 weeks than those at 6 weeks and baseline in both exercise groups.

Conclusions Aerobic exercise training and resistance exercise training can change levels of myokines and improve body composition and physical fitness of obese females. These findings provide preliminary evidence that obese females need to exercise or perform physical activity to improve their levels of myokines and physical fitness.

\section{Background}

The human body consists of about 600 muscles that account for about $40 \%-50 \%$ of total body weight in non-obese adults [1]. Skeletal muscles can adapt to mechanical, nerve, and humoral stimulation. They play an important role in physical activity, energy consumption, and glucose elimination in all humans [2]. The concept that skeletal muscles can secrete humoral elements to actively communicate with other organs has been proposed several years ago [3, 4]. Henningsen et al. [5] and Pedersen et al. [6] have used the term of "myokines" to describe cytokines and other peptides that are expressed and released by muscle cells.

During exercise or physical activity, myokines, cytokines, and other peptides are secreted by work out muscles within the muscle tissue or in an endocrine by targeting distant organs [7]. In response to muscle contraction, skeletal muscle can circulate to express and release myokines. More than one hundred myokines have been identified, including interleukin-6 (IL-6), interleukin-15 (IL-15), brain-derived neurotrophic factor (BDNF), insulin-like growth factor (IGF1), fibroblast growth factor 2 (FGF2), fibroblast growth factor 21 (FGF21), follistatin-related protein 1 (FSTL1), irisin, and so on [8].

Previous studies have reported that physical activity can significantly increase circulating levels of skeletal muscle-derived myokines (i.e., IL-6) that can trigger beneficial changes in circulating levels of 
several other inflammatory mediators [9]. It has been reported that levels of IL-15 mRNA as a kind of myokine are upregulated in human skeletal muscles following a bout of strength training in young males [10]. BDNF mRNA and protein expression levels are also increased in skeletal muscles of untrained males after acute aerobic exercise [11].

Several studies have reported effects of exercise on myokines. Moreover, it is well-known that exercise or physical activity can decrease body weight in the obesity population. However, there are some studies that shows that myokines review and short term intervention studies. In addition, the association between myokines and obese adults with regular exercise or physical activity has not been previously reported. Therefore, the purpose of this study was to investigate changes in levels of myokines after regular aerobic training and resistance training in post- menopausal females with obesity.

\section{Methods}

Study participation

A total of 41 post- menopausal obese females volunteered to participate in this study who performed regular physical exercise (aerobic exercise and resistance exercise). These participants were randomly assigned to one aerobic exercise group $(n=21)$ and one resistance exercise group $(n=20)$. To be included in the present study, participants had to meet the following inclusion criteria: 1) post- menopausal (absence of a menstrual cycle for at least 3 months) on the date of the assessment; 3 ) not receiving hormone replacement treatment; and 4) not using drugs such as beta-blockers, statins, and so on. The sample size of the subjects was calculated by using ANOVA a large size of effect size of .90, a significance level of .05 and a power of .80 (G*power 3.1.2). The results of sample size per group were calculated as 15 people each group.

These participants did not participate in a regular structured resistance or an aerobic exercise program for at least four months prior to this study. All subjects who agreed to participate in this study had the study explained to them to ensure a complete understanding of its purpose and methods used. This study was performed following the ethical standard of the Declaration of Helsinki. Subjects signed an informed consent form before participation. This study was approved by Kangwon National University Review Board for Human Subjects (KWNUIRB-2016-04-009-002).

Physical characteristics of participants are shown in Table 1. 
Table 1

Characteristic of all participants

\begin{tabular}{|llll|}
\hline Variable & Resistance exercise $(\mathbf{n}=\mathbf{2 0})$ & Aerobic exercise $(\mathbf{n}=\mathbf{2 1})$ & p-value \\
\hline Age (years) & $52.50 \pm 7.65$ & $56.67 \pm 5.43$ & 0.051 \\
\hline Height $(\mathrm{cm})$ & $157.89 \pm 4.30$ & $155.67 \pm 5.96$ & 0.180 \\
\hline Weight $(\mathrm{kg})$ & $62.82 \pm 10.09$ & $62.06 \pm 9.19$ & 0.801 \\
\hline BMI $\left(\mathrm{kg} / \mathrm{m}^{2}\right)$ & $25.16 \pm 3.67$ & $25.67 \pm 3.67$ & 0.655 \\
\hline$\%$ fat $(\%)$ & $36.02 \pm 5.87$ & $36.53 \pm 5.89$ & 0.781 \\
\hline Muscle mass $(\mathrm{kg})$ & $21.60 \pm 3.00$ & $21.26 \pm 2.98$ & 0.719 \\
\hline SBP $(\mathrm{mmHg})$ & $129.60 \pm 18.29$ & $129.24 \pm 16.38$ & 0.947 \\
\hline DBP (mmHg) & $81.00 \pm 8.69$ & $82.00 \pm 9.95$ & 0.734 \\
\hline $\begin{array}{l}\text { Values are mean } \pm \text { SD. } \\
\text { BMl: body mass index; } \text { SBP: systolic blood pressure; DBP: diastolic blood pressure. }\end{array}$ \\
\hline
\end{tabular}

[Table 1. Characteristic of subjects]

Measurement of body composition

Physical and anthropometric variables were measured for both groups. Body mass and height were measured to the nearest $0.1 \mathrm{~kg}$ and $0.1 \mathrm{~cm}$, respectively, using a body composition analyzer (Inbody 720, Body Composition Analyzer; Biospace, Seoul, Korea). Body mass index (BMI) was calculated as weight in kilograms divided by the square of height in meters.

Measurement of physical fitness test

Physical fitness variables were measured at baseline, 6 weeks, and 12 weeks in both exercise groups. In the evaluation of physical fitness, we utilized muscle strength, muscle endurance, flexibility, power, and agility. Grip strength was used to assess muscle strength, which measured with a digital grip dynamometer (GRIP-D 5101; TAKEI, Co., Japan). The legs are slightly spread and the arms opened in a natural way. After straightening the arms at about $15^{\circ}$ and keeping the torso upright, the dynamometer can be grabbed. Sit-up was used to assess muscle endurance, which measuring device with both hands crossed over the head and the abdominal strength used to raise the upper body for 30 seconds was measured. Sit and reach were used to assess flexibility, which measuring instrument with a posture of 90 degrees at the waist and the knees bent forward without reaching the bend. The distance $(\mathrm{cm})$ at which the fingertip was pushed out was measured. Side-step was used to assess agility, which measured with a side step meter, with both feet spread out around the center line. We moved to the step with the start at both sides at a $100 \mathrm{~cm}$ interval, and one foot returned to the first posture beyond the right (or left) line. The number of times when both feet moved around the center line for 20 seconds was measured. Vertical 
jump was used to assess power, which vertical jump-measuring instrument on a mat, taking a ready position and jumping with the start. At that time, the knee was not lifted into the air, and the highest value from two trials was recorded. All tests were performed twice and the best score was retained.

\section{Experimental exercise program}

The 12-week exercise program intervention consisted of three days of aerobic exercise and resistance exercise per week (i.e., Monday, Wednesday, and Friday) in each group. The resistance exercise group had 10 minutes of warm-up activities, 40 minutes of the main resistance exercise program, and 10 minutes of cool-down. The intensity was set at 55 65\% of the one-repetition maximum (1RM) with one minute of rest period between all sets and a 1-minute rest between different exercises. Main resistance exercise was trained by squat and lunge for lower, chest press and vertical fly for chest, let pull-down and long pull for back, and crunch for abdominal. The aerobic exercise group had 10 minutes of warm-up activities, 30 minutes of the main aerobic exercise program, and 10 minutes of cool-down. When aerobic exercise was performed on a treadmill, the intensity was set at $50 \sim 60 \%$ of the heart rate max (HRmax) via continuous monitoring of heart rates (Polar M400; Polar H7 WearLink; Polar Electro Oy, Kempele, Finland).

A familiarization session took place during the first week. Participants were instructed to learn how to perform each resistance exercise in a safe way. Prior to participation, all subjects were informed of the risks associated with this research study. After obtaining informed consent, subjects completed questionnaires and pre-testing prior to participate in this study. They were familiarized with study procedures during the week prior to implementation of each exercise program.

Blood collection and myokines analysis

Fasting venous blood samples were collected from all participants at baseline, 6 weeks, and 12 weeks. Fasting was maintained for $12 \mathrm{~h}$. Blood samples were collected on the following day. Enough sleep and radical movement as much as possible to refrain. All samples were taken at 08:30 AM from antecubital veins. The samples were immediately centrifuged at $3,500 \mathrm{~g}$ at $4{ }^{\circ} \mathrm{C}$ for $10 \mathrm{~min}$, after which serum was stored in $-80^{\circ} \mathrm{C}$. until further analysis. Serum levels of myokines (IL-6, IL-15, and BDNF) were measured using enzyme-linked immunosorbent assay Dueset kits (R\&D systems, Minneapolis, MN, USA) according to the manufacturer's instructions as described previously.

\section{Statistical analysis}

All results are reported as the mean \pm standard deviation. All data were analyzed using SPSS version 25.0 (SPSS Inc., Chicago, IL, USA). First, we used an independent samples t-test to assess group differences in baseline variables. For the two groups (resistance exercise vs. aerobic exercise) by three stages (baseline, 6 weeks, and 12 weeks), two-way within-subject factor ANOVA was used to examine whether exercise type and time influenced physical fitness and myokines. Bonferroni test was used for post-hoc analysis. The normality of dependent variables was determined by Shapiro-Wilk tests. Statistical significance was accepted at $\mathrm{p}<0.05$. 


\section{Results}

Change of body composition after training

Changes of body composition in each group are shown in Table 2. Two-way within-factor ANOVA revealed significant $(\mathrm{p}<0.05)$ group $\times$ time interaction for body mass index $(\mathrm{BMI})$. Post-hoc analysis using Bonferroni test indicated that weight, \% fat, BMI, WHR, and SBP were significantly decreased at 12 weeks than those at 6 weeks and baseline in the resistance exercise group. In addition, weight, BMI, and \% fat were greater at 12 weeks than those at 6 weeks and baseline in the aerobic exercise group. 
Table 2

Measurements of each body composition by group and time

\begin{tabular}{|c|c|c|c|c|}
\hline \multirow[t]{2}{*}{ Variable } & \multirow[t]{2}{*}{ time } & \multicolumn{2}{|l|}{ Type of exercise } & \multirow{2}{*}{$\begin{array}{l}\text { p-value } \\
\text { (interaction) }\end{array}$} \\
\hline & & Resistance exercise $(n=20)$ & Aerobic exercise $(n=21)$ & \\
\hline \multirow[t]{3}{*}{ Weight (kg) } & Pre & $62.82 \pm 10.09 a^{a^{*}}$ & $62.06 \pm 9.19 a^{a^{*}}$ & \multirow[t]{3}{*}{0.050} \\
\hline & Mid & $62.57 \pm 9.70$ & $60.90 \pm 9.00^{b^{\star *}}$ & \\
\hline & Post & $61.58 \pm 8.92 \mathrm{c}^{\star}$ & $60.69 \pm 9.24$ & \\
\hline \multirow[t]{3}{*}{$\mathrm{BMI}\left(\mathrm{kg} / \mathrm{m}^{2}\right)$} & Pre & $25.16 \pm 3.67 \mathrm{a}^{*}$ & $25.67 \pm 3.67^{a^{\star \star}}$ & \multirow[t]{3}{*}{0.024} \\
\hline & Mid & $25.12 \pm 3.68$ & $25.15 \pm 3.58 b^{b^{\star \star \star}}$ & \\
\hline & Post & $24.69 \pm 3.29 \mathrm{c}^{\star}$ & $25.07 \pm 3.70$ & \\
\hline \multirow[t]{3}{*}{$\%$ fat $(\%)$} & Pre & $36.02 \pm 5.87^{a^{* *}}$ & $36.53 \pm 5.89 a^{a^{* \star *}}$ & \multirow[t]{3}{*}{0.983} \\
\hline & Mid & $34.52 \pm 6.09^{b^{*}}$ & $34.90 \pm 5.55^{b^{\star \star \star}}$ & \\
\hline & Post & $33.88 \pm 5.17$ & $34.30 \pm 5.28^{c^{*}}$ & \\
\hline \multirow[t]{3}{*}{ Muscle mass (kg) } & Pre & $21.60 \pm 3.00^{a^{*}}$ & $21.26 \pm 2.98$ & \multirow[t]{3}{*}{0.295} \\
\hline & Mid & $22.10 \pm 3.10$ & $21.34 \pm 3.01$ & \\
\hline & Post & $22.02 \pm 3.17$ & $21.47 \pm 3.15$ & \\
\hline \multirow[t]{3}{*}{ WHR } & Pre & $0.91 \pm 0.05^{a^{*}}$ & $0.91 \pm 0.05 \mathrm{a}^{\star \star}$ & \multirow[t]{3}{*}{0.519} \\
\hline & Mid & $0.89 \pm 0.05$ & $0.89 \pm 0.04$ & \\
\hline & Post & $0.89 \pm 0.04 \mathrm{c}^{*}$ & $0.89 \pm 0.04$ & \\
\hline \multirow[t]{3}{*}{$\mathrm{SBP}(\mathrm{mmHg})$} & Pre & $129.6 \pm 18.3^{a^{*}}$ & $129.2 \pm 16.4$ & \multirow[t]{3}{*}{0.987} \\
\hline & Mid & $130.8 \pm 16.1$ & $130.3 \pm 15.9$ & \\
\hline & Post & $122.6 \pm 19.3^{c^{\star \star}}$ & $122.7 \pm 11.7^{\mathrm{c}^{\star}}$ & \\
\hline $\mathrm{DBP}(\mathrm{mmHg})$ & Pre & $81.00 \pm 8.69$ & $82.00 \pm 9.95$ & 0.636 \\
\hline \multicolumn{5}{|c|}{ Values are expressed as Mean \pm SD. } \\
\hline \multicolumn{5}{|c|}{$\begin{array}{l}\text { BMI: body mass index; SBP: systolic blood pressure; DBP: diastolic blood pressure; Pre: baseline; Mid: } \\
6 \text { weeks; Post: } 12 \text { weeks. }\end{array}$} \\
\hline \multicolumn{5}{|c|}{ A: pre vs. post; b: pre vs. mid; c: mid vs. post. } \\
\hline$\star p<0.05, * * p<0.0$ & *** & 0.001 & & \\
\hline
\end{tabular}




\begin{tabular}{lll} 
Mid & $84.40 \pm 10.80$ & $85.90 \pm 9.43$ \\
\hline Post & $80.00 \pm 11.01^{\mathrm{c}^{\star \star}}$ & $79.14 \pm 10.22^{\mathrm{c}^{*}}$
\end{tabular}

Values are expressed as Mean \pm SD.

BMI: body mass index; SBP: systolic blood pressure; DBP: diastolic blood pressure; Pre: baseline; Mid: 6 weeks; Post: 12 weeks.

A: pre vs. post; b: pre vs. mid; c: mid vs. post.

$* p<0.05, * * p<0.01, * \star * p<0.001$

[Table 2. Measurements of each body composition by group and time]

Change of physical fitness after training

Changes in physical fitness in each group are shown in Table 3 . There was no significant group $\times$ time interaction. Post-hoc analysis using Bonferroni test indicated that muscle endurance, power, and agility in the resistance exercise group were significantly increased at 12 weeks than those at 6 weeks and baseline. In the aerobic exercise group, muscle strength, flexibility, muscle endurance, and agility were also greater at 12 weeks than those at 6 weeks and baseline. 
Table 3

Measurements of each physical fitness by group and time

\section{Please click here to download the \\ original image file \\ Click here to Correct}

\begin{tabular}{llllll} 
Variable & time & \multicolumn{2}{c}{ Type of exercise } & pevalue \\
\cline { 2 - 4 } & & $\begin{array}{l}\text { Resistance } \\
\text { exercise }(\mathrm{n}=20)\end{array}$ & $\begin{array}{l}\text { Aerobic } \\
\text { exercise }(\mathrm{n}=\end{array}$ & \\
(interaction)
\end{tabular}

Values are expressed as Mean \pm SD.

Pre: baseline; Mid: 6 weeks; Post: 12 weeks.

A: pre vs. post; b: pre vs. mid; c: mid vs. post.

${ }^{\star} p<0.05,{ }^{* \star} p<0.01, * \star * p<0.001$ 


\section{Please click here to download the}

\section{original image file}

Click here to Correct

Please click here to download the original

image file

Click here to Correct

Please click here to download the original

image file

Click here to Correct

Figure 1. Levels of myokines by group and time

Pre: baseline; Mid: 6 weeks; Post: 12 weeks.

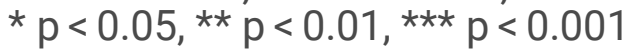

Values are expressed as Mean \pm SD.

Pre: baseline; Mid: 6 weeks; Post: 12 weeks.

A: pre vs. post; b: pre vs. mid; c: mid vs. post.

$\star p<0.05, * \star p<0.01, * \star * p<0.001$

[Table 3. Measurements of each physical fitness by group and time]

Change in levels of myokines after training

[Fig. 1. Measurements of each myokines by group and time]

\section{Discussion}

In this study, changes in physical fitness and levels of myokines levels after aerobic exercise training and resistance exercise training in obese females were investigated. The main finding of this study was that levels of myokines were significantly increased after exercise training in both groups. In addition, weight, $\mathrm{BMI}$, and \% fat were significantly decreased after exercise training in both groups. Muscle mass was significantly increased only in the resistance exercise training group. Regarding physical fitness, muscle endurance and agility were significantly increased after exercise training in both groups.

In females, particular post- menopausal females were decrease in muscle fibers [12]. And this phase female was dynamic change sex hormone may increase body fat, that cause by obesity, type 2 diabetes, cardiovascular disease, and other chronic diseases [13]. Therefore, post- menopausal females found paradoxical associations of BMl; overweight/obesity was associated [14]. In this study participants were obese (more than $30 \%$ fat) and post- menopausal. Regular exercise (physical activity) induces metabolism and mitochondrial adaptation, improving energy metabolism and the function of many organs [15]. The skeletal muscle is the largest organ in the human body. Several years ago, a volumetric factor (i.e., cytokine) produced and released from muscle cell contraction has been identified [16]. It 
appeared to have a large metabolic effect. Its contraction as a cytokine-producing organ has revealed an entirely new paradigm showing that the skeletal muscle is an endocrine organ that can affect the metabolism of tissues and organs by secreting factors such as hormones [17]. Although adipose tissue has been considered a major source of cytokines (i.e., adipokines), muscles can also produce and release myokines. This suggests that, in addition to adipose tissue, skeletal muscle can also be a major source of molecules secreted [18]. An obese person with post- menopausal has abundant adipose tissues that can secrete adipokines known to induce a pathogenic and pro-inflammatory environment [19]. This is the reason why obesity increases the secretion of cytokines. Thus, a post- menopausal obese person is exposed to various diseases. In this study, we observed changes in levels of myokines after 6 weeks and 12 weeks of aerobic exercise training or resistance exercise training in obese females who postmenopausal. Results revealed that levels of myokines such as IL-6, IL-15, and BDNF were significantly increased after exercise training in both groups.

The circulation level of IL- 6 is affected by the duration and intensity of human muscle contractions [20] (Helge et al., 2003). IL-6 increases glucose absorption and fatty acid oxidation in vitro through AMP-active protein kinase and PI3K-Akt signal pathways [21]. In addition, muscle-derived IL- 6 can inhibit low TNF-a (tumor-necrosis factor-alpha) production, thereby inhibiting TNF-a-induced insulin resistance. Therefore, it may be important to mediate beneficial health effects of exercise [22]. As a kind of myokine, interleukin15 (IL-15) can inhibit adipose tissue deposition in both laboratory animals and human subjects [23]. The complex regulation of IL-15 expression and secretion has been summarized [23]. IL-15 also exhibits proinflammatory and anti-inflammatory action in various tissues. It has both a positive anticancer effect through stimulation of NK cells and a deleterious effect associated with inflammatory bowel disease [24]. Brain-derived neurotrophic factor (BDNF) is known to be released mainly in the hypothalamus. It is a key factor that regulates nerve development, plasticity, and energy homeostasis [25]. It is interesting to note that gene and protein expression levels of BDNF are upregulated in human skeletal muscle after exercise [26]. Previous studies have reported that 12 weeks of resistance training can significantly increase levels of BDNF, but not levels of IL-15 [27]. Eaton et al. [28] have reported that IL-6 levels are significantly increased after a high-intensity interval exercise. Banitalebi et al. [29] have shown that combined exercise conducted for 10 weeks can significantly decrease levels of IL-6 and IL-15 in overweight women with type II diabetes. We observed that levels of IL-6, IL-15, and BDNF were significantly increased after exercise training in both groups (aerobic exercise and resistance exercise). Myokines contribute to autocrine regulation of metabolism in the muscle itself and paracrine/endocrine regulation of other adjacent/remote organs [30]. BDNF and IL- 6 are involved in AMPK-mediated fat oxidation. IL-6 and IL-15 also stimulate lipolysis of visceral fat [31]. It is known that exercise training requires more muscle contraction.

In this study, participants were obese (over $30 \%$ fat). They had more adipose tissues than the normal weight population. Body weight, $\mathrm{BMI}$, and \% fat were significantly decreased after exercise than those at baseline. Levels of myokines were also changed after exercise. There was a significant increase in muscle mass in the resistance training group, but not in the aerobic training group. Changes in muscle mass might be due to differences in characteristics between aerobic and anaerobic exercise. Moreover, 
changes in body composition might have affected changes in physical fitness of obese women. The resistance training group showed significant improvements in muscle endurance, power, and agility, while the aerobic training group showed significant improvements in muscle strength, muscle endurance, and agility. Physical fitness and physical activity or exercise training are well-known to have benefits for the health of the obesity population [32]. Many recent studies have shown that resistance exercise training has a similar effect to aerobic exercise training, including a positive effect on obesity [33,34]. Most previous studies support regular exercise because it can increase in muscle mass but decrease weight loss and \% fat. The increase of myokines due to contraction of the large muscles and loss of body weight might have improved physical fitness.

The present study has some limitations. The age of participants in this study had a large variation. Although the age difference between the two groups was not significant, subsequent studies with smaller age difference are needed. Another limitation was that the sample size was relatively small. Further studies with larger populations are needed to validate our findings.

\section{Conclusion}

In conclusion, this study indicates that aerobic exercise training and resistance exercise training can change levels of myokines and improve body composition and physical fitness in obese females who post- menopausal. These findings provide preliminary evidence that post- menopausal obese females need to perform exercise or physical activity to improve their levels of myokines and physical fitness.

\section{Abbreviations}

\section{IL-6}

interleukin-6; IL-15:interleukin-15; BDNF:brain-derived neurotrophic factor; IGF-1:insulin-like growth factor; FGF2:fibroblast growth factor 2; FGF21:fibroblast growth factor 21; FSTL1:follistatin-related protein 1; BMI:body mass index; WHR:waist-hip ratio; RM:repetition maximum; HR:heart rate; SPSS:statistical package for social science; ANOVA:analysis of variance; AMP:adenosine monophosphate; TNF-a:tumornecrosis factor-alpha; AMPK:activated protein kinase;

\section{Declarations}

\section{Acknowledgements}

We thank all the study participants and staff for their assistance.

\section{Authors' contributions}

Sunghwun Kang and Seung-Taek Lim contributed to conception and design of the study. 
Sunghwun Kang and Seung-Taek Lim implemented the measurements and training sessions. II Bong Park analysed the participant data. All authors interpreted and discussed the results. All authors drafted parts of the manuscript. All authors read and approved the final manuscript

\section{Funding}

Not applicable.

\section{Availability of data and materials}

Full data for this research is available through the corresponding author upon request.

\section{Ethics approval and consent to participate}

The study was approved by Kangwon National University Institutional Review Board, and conducted in agreement with the Declaration of Helsinki. In advance of their participation, all of the participants were fully informed about the purpose and experimental procedures of the study. All of the participants completed consent forms. The participants were informed that all data collected would be processed anonymously.

\section{Consent for publication}

Not applicable.

\section{Competing interests}

The authors declare that they have no competing interests.

\section{Author details}

${ }^{1}$ Laboratory of Exercise Physiology, Department of Sport Science, College of Art and Culture, Kangwon National University, Gangwon-do, Republic of Korea. ${ }^{2}$ Department of Sport Industry, Busan University of Foreign Studies, Busan, Republic of Korea. ${ }^{3}$ Institute of Sport Science, Kangwon National University, 
Gangwon-do, Republic of Korea. ${ }^{4}$ Waseda Institute for Sport Sciences, Waseda University, Saitama, Japan. ${ }^{5}$ Nasaret International Hospital, Incheon, Republic of Korea

\section{References}

1. Schnyder S, Handschin C. Skeletal muscle as an endocrine organ: PGC-1a, myokines and exercise. Bone. 2015;80:115-125.

2. Turner N, Cooney GJ, Kraegen EW, Bruce CR. Fatty acid metabolism, energy expenditure and insulin resistance in muscle. J Endocrinol. 2014;220:T61-79

3. Hawley JA, Hargreaves M, Joyner MJ, Zierath JR. Integrative biology of exercise. Cell. 2014;159:73849.

4. Giudice J, Taylor JM. Muscle as a paracrine and endocrine organ. Curr Opin Pharmacol. 2017;34:4955.

5. Henningsen J, Rigbolt KT, Blagoev B, Pedersen BK, Kratchmarova I. Dynamics of the skeletal muscle secretome during myoblast differentiation. Mol Cell Proteomics. 2010;9:2482-96.

6. Pedersen BK, Febbraio MA. Muscles, exercise and obesity: skeletal muscle as a secretory organ. Nat Rev Endocrinol. 2012;8:457-65.

7. Pedersen L, Hojman P. Muscle-to-organ cross talk mediated by myokines. Adipocyte. 2012;1:164-7.

8. Díaz BB, González DA, Gannar F, Pérez MCR, de León AC. Myokines, physical activity, insulin resistance and autoimmune diseases. Immunol Lett. 2018;203:1-5.

9. Nimmo MA, Leggate M, Viana JL, King JA. The effect of physical activity on mediators of inflammation. Diabetes Obes Metab. 2013;15 Suppl 3:51-60.

10. Nielsen AR, Mounier R, Plomgaard P, Mortensen $\mathrm{OH}$, Penkowa M, Speerschneider T, et al. Expression of interleukin-15 in human skeletal muscle effect of exercise and muscle fibre type composition. $J$ Physiol. 2007;584(Pt 1):305-12.

11. Matthews VB, Aström MB, Chan MH, Bruce CR, Krabbe KS, Prelovsek O, et al. Brain-derived neurotrophic factor is produced by skeletal muscle cells in response to contraction and enhances fat oxidation via activation of AMP-activated protein kinase. Diabetologia. 2009;52:1409-18.

12. Aubertin-Leheudre M, Lord C, Goulet ED, Khalil A, Dionne IJ. Effect of sarcopenia on cardiovascular disease risk factors in obese postmenopausal women. Obesity (Silver Spring). 2006;14:2277-83.

13. Cauley JA, Gutai JP, Kuller LH, LeDonne D, Powell JG. The epidemiology of serum sex hormones in postmenopausal women. Am J Epidemiol. 1989;129:1120-31.

14. Tanaka S, Kuroda T, Saito M, Shiraki M. Overweight/obesity and underweight are both risk factors for osteoporotic fractures at different sites in Japanese postmenopausal women. Osteoporos Int. 2013;24:69-76.

15. Drake JC, Wilson RJ, Yan Z. Molecular mechanisms for mitochondrial adaptation to exercise training in skeletal muscle. FASEB journal. 2016;30:13-22. 
16. Peake JM, Della Gatta P, Suzuki K, Nieman DC. Cytokine expression and secretion by skeletal muscle cells: regulatory mechanisms and exercise effects. Exerc Immunol Rev. 2015;21:8-25.

17. Pedersen BK. Muscles and their myokines. J Exp Biol. 201;214(Pt 2):337-46.

18. Bleau C, Karelis AD, St-Pierre DH, Lamontagne L. Crosstalk between intestinal microbiota, adipose tissue and skeletal muscle as an early event in systemic low-grade inflammation and the development of obesity and diabetes. Diabetes Metab Res Rev. 2015;31:545-61.

19. Palmer BF, Clegg DJ. The sexual dimorphism of obesity. Mol Cell Endocrinol. 2015;402:113-9.

20. Helge JW, Stallknecht B, Pedersen BK, Galbo H, Kiens B, Richter EA. (2003) The effect of graded exercise on IL-6 release and glucose uptake in human skeletal muscle. J Physiol. 2003;546(Pt 1):299305.

21. Coles CA. Adipokines in Healthy Skeletal Muscle and Metabolic Disease. Adv Exp Med Biol. 2016;900:133-60.

22. Pedersen BK, Fischer CP. Beneficial health effects of exercise-the role of IL- 6 as a myokine. Trends Pharmacol Sci. 2007;28:152-6.

23. Quinn LS, Anderson BG. Interleukin-15, IL-15 Receptor-Alpha, and Obesity: Concordance of Laboratory Animal and Human Genetic Studies. J Obes. 2011;2011:456347.

24. Dozio E, Malavazos AE, Vianello E, Briganti S, Dogliotti G, Bandera F. Interleukin-15 and soluble interleukin-15 receptor a in coronary artery disease patients: association with epicardial fat and indices of adipose tissue distribution. PLoS One. 2014;9:e90960.

25. Shibata A, Hanatani A, Izumi Y, Kitada R, Iwata S, Yoshiyama M. Serum brain-derived neurotrophic factor level and exercise tolerance complement each other in predicting the prognosis of patients with heart failure. Heart Vessels. 2018;33:1325-33.

26. Pedersen BK, Pedersen M, Krabbe KS, Bruunsgaard H, Matthews VB, Febbraio MA. Role of exerciseinduced brain-derived neurotrophic factor production in the regulation of energy homeostasis in mammals. Experimental physiology. 2009;94:1153-60.

27. Urzi F, Marusic U, Ličen S, Buzan E. Effects of Elastic Resistance Training on Functional Performance and Myokines in Older Women-A Randomized Controlled Trial. J Am Med Dir Assoc. 2019;20:830-4.

28. Eaton M, Granata C, Barry J, Safdar A, Bishop D, Little JP. Impact of a single bout of high-intensity interval exercise and short-term interval training on interleukin-6, FNDC5, and METRNL mRNA expression in human skeletal muscle. J Sport Health Sci. 2018;7:191-6.

29. Banitalebi E, Kazemi A, Faramarzi M, Nasiri S, Haghighi MM. Effects of sprint interval or combined aerobic and resistance training on myokines in overweight women with type 2 diabetes: $A$ randomized controlled trial. Life sciences. 2019;217:101-9.

30. Kim S, Choi JY, Moon S, Park DH, Kwak HB, Kang JH. (2019) Roles of myokines in exercise-induced improvement of neuropsychiatric function. Pflügers Archiv. 2019;471: 491-505.

31. Pedersen BK. Anti-inflammatory effects of exercise: role in diabetes and cardiovascular disease. European journal of clinical investigation. 2017;47:600-11. 
32. Mora-Gonzalez J, Esteban-Cornejo I, Cadenas-Sanchez C, Migueles JH, Rodriguez-Ayllon M, MolinaGarcía P, et al. Fitness, physical activity, working memory, and neuroelectric activity in children with overweight/obesity. Scand J Med Sci Sports. 2018;29:1352-63.

33. Francois ME, Durrer C, Pistawka KJ, Halperin FA, Little JP. Resistance-based interval exercise acutely improves endothelial function in type 2 diabetes. American journal of physiology. Heart and circulatory physiology. 2016;311:H1258-67

34. Talebi-Garakani E, Safarzade A. Resistance training decreases serum inflammatory markers in diabetic rats. Endocrine. 2013;43:564-70.

\section{Figures}

\section{Change of nyokines after training}
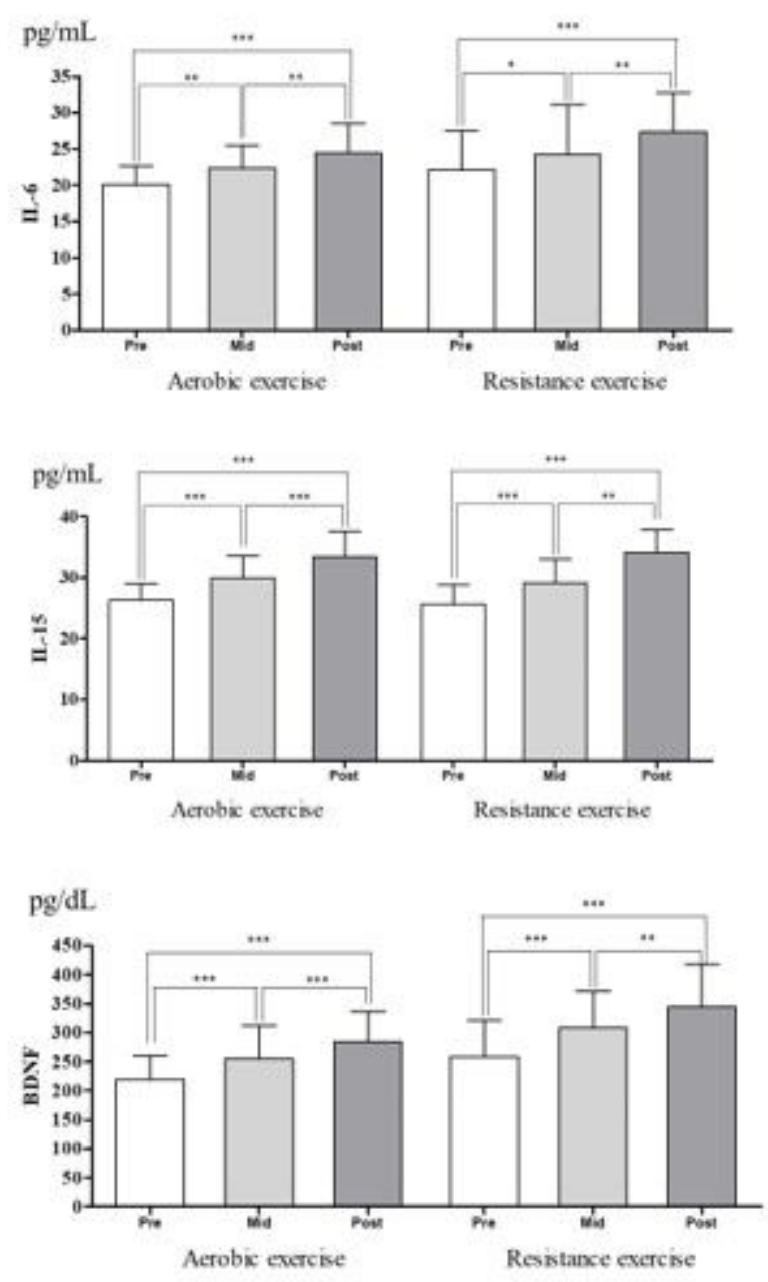

\section{Figure 1}

Levels of myokines by group and time Pre: baseline; Mid: 6 weeks; Post: 12 weeks. ${ }^{*} p<0.05,{ }^{* \star} p<0.01$, $\star \star * * 0.001$ 